\title{
Primary Exploring of POGIL-MOOC Combined Teaching Mode for Medical Graduate Education
}

\author{
Hong Zhang ${ }^{1, ~ a, ~ Y u t i n g ~ F a n ~}{ }^{2, b}$ and Yang Luo ${ }^{1, c}$ \\ ${ }^{1}$ Medical Research Center, Southwest Hospital, Third Military Medical University, Chongqing, \\ 400038, China; \\ ${ }^{2}$ Foreign language school affiliated to Sichuan Foreign Language University, Chongqing, 400039, \\ China

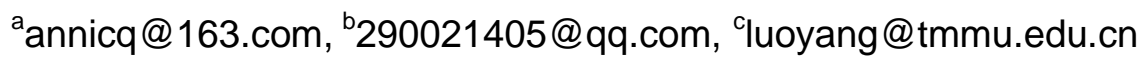

Keywords: POGIL; MOOC; Educational reform; Innovative ability

\begin{abstract}
Edical graduate education is an activity of teaching and learning to create and apply profound professional knowledge. However, current medical graduate education is following an undergraduate model, where guided teaching and hubristic teaching is scarce, curriculum is lagging, the activity of students is not high, and students lack the ability to think independently and to conduct creative science work. Therefore, there is an imperative requirement of new medical graduate education model that adapts to modern "industry-university-research" integration. This article will apply process oriented guided inquiry learning (POGIL) mode combined with massive online open classes (MOOC) to medical graduate education teaching mode reform, analyze the feasibility and strategy of the combination of the two modes. Meantime, we explored the facing problems and challenges from the combination, performing a strategic research of POGIL-MOOC combined application to improve the creative ability of medical graduates and providing powerful support for medical graduate education to cultivate innovative experts.
\end{abstract}

\section{“POGIL” 联合 “MOOC” 教学的研究生培养模 式初探}

\author{
张洪 ${ }^{1, a}$, 樊玉婷 ${ }^{2, \mathrm{~b}}$, 罗阳 ${ }^{1, \mathrm{o}}$ \\ 1. 第三军医大学第一附属医院综合实验研究中心, 中国重庆 400038 \\ 2. 四川外国语大学附属外国语学校, 中国 重庆 400039 \\ aannicq@163.com, b290021405@qq.com, cluoyang@tmmu.edu.cn
}

摘要: 医学研究生教育是创造和应用高深专门知识的教与学的活动。然而, 目前医学研究生 教育 “本科化”, 启发式教学甚少, 课程设置滞后, 学生缺乏独立思维能力, 尤其是科研创 新能力。因此, 亟需一种适应现代 “产学研”一体化要求的新型医学研究生教育模式。本文 将面向过程的指导型探究学习 (POGIL) 结合慕课 (MOOC) 教学用于医学研究生培养, 分 析其二者联合运用的可行性、策略以及存在的挑战, 从而进行了医学研究生创新能力培养的 模式改革初探, 为研究生教育尤其是医学研究生教育提供有力支撑。

关键词: POGIL; MOOC; 教学改革; 创新能力 


\section{1. 引言}

研究生教育是创造和应用高深专门知识的教与学的活动。然而, 在知识经济时代, 研究生扩 招成为一种普遍的国际现象, 在增量式发展的驱动下, 研究生教育正面临着质量下降、创新 不足和供求失衡等突出问题 [1]。随着医学技术向精准医疗的发展, 对艾滋病、癌症等疾病的 发病机理尚未清楚, 多种疾病的诊断及治疗等尚存在困难, 这就对医学研究生创新思维培养 提出了更高的要求 [2]。现实的种种问题无不挑战着传统过于单一的医学研究生教育模式, 亟 需探索适应时代发展的医学研究生的新型培养模式。

面向过程的指导型探究学习 (Process Oriented Guided Inquiry Learning, POGIL) 是一种融合学 习过程与学习结果的教学理念和方法, 在注重基本概念掌握的同时, 鼓励学生深度思考和培 养创新思维 [3]。然而, POGIL 教学主要集中在研讨的几十分钟内, 思维的开发是有限的, 在课后对学生积极性的提高依然是比较缺乏的。慕课 (Massive Open Online Course, MOOC) 于 2012 年兴起后受到教育界热捧, 是一种大规模在线开放课的教学新模式, 其 “精品化”、 “碎片化”、“移动化” 课程内容, 可以在课后充分调动学生主动性、积极性 [4]。美国各大 名校纷纷开设了自己的慕课课程, 并形成了 Coursera, edX, Udacity 和 Udemy 四大慕课平台 [5]。

目前, POGIL 模式主要应用于药学、物理化学、计算机以及市场营销等课程的教学过程中, 而将 POGIL 结合 MOOC 模式应用于检验医学的研究生的培养尚未见相关报导 [6-7]。本文将 POGIL 与 MOOC 联用, 进行检验医学研究生教学模式改革初探, 以提升检验医学研究生基本 技能及科研思维能力, 以期为检验医学研究生乃至整个医学研究生培养探索一种新型的教学 模式。

\section{POGIL 结合 MOOC 教学模式促进医学研究生创新能力培养}

与医学本科教育面对经加工的系统化、结构化知识不同, 医学研究生教育面对的是不确定性 的原生态知识, 这就决定了学习者必须具备抽象性和复杂性的高级认识和思维水平 [8]。相对 而言, 我国的医学研究生课程大部分依然沿袭本科生时期的以教师课堂讲授为主的注入式教 学, 教师组织引导下的启发式教学和引导式教学甚少。研究生教育 “本科化”导致培养方案长 期不变, 课程设置落后, 学生积极性不高, 缺乏独立思维能力, 尤其是科研创新能力。衡量 研究生教育发展水平的重要标志是它在知识创新方面的贡献, 纯粹以传授知识为导向的研究 生教育必然会阻碍医学的发展。

POGIL 教学注重培养学生学习技能, 包括信息加工处理, 批判性思维能力, 主动解决问题能 力, 合作能力和元认知能力 [9]。这种模式改变了传统以教师讲授为核心的教学模式, 学生从 单纯被动的接受者转变成积极的科学思想家, 有效的激发学生的创造力 [10]。这无形中与研 究生要求具备抽象性和复杂性的高级认识和思维水平不谋而合。在短短几年时间内, 培养医 学研究生成长为具有创新思维的 “产学研” 一体化的新时代医务及科研工作者, 时间紧, 任 务重。MOOC 碎片化的学习模式正好弥补这一缺陷 [11]。与传统的网络精品课程不同, 慕课 都是以微视频的形式制作的, 设计者对知识点进行分解提炼后, 制作成一个个时长仅 3-10 分 钟的慕课微视频。在 POGIL 中引入 MOOC 教学进一步使研究生在有限的时间内, 不仅可以 多元化的开展学习与探索, 促进 POGIL 教学中关键知识结构的掌握, 更可以了解国内外先进 技术, 共享世界先进知识成果; 同时适应数字化时代碎片化学习的特点, 方便研究生自主选 择, 真正实现了随时随地学习, 帮助医学研究生培养终身学习的习惯 [12]。因此 POGIL 结合 MOOC 应用于医学研究生创新能力的培养具有其特有的优势, 是医学研究生教育改革中值得 探索的道路。 


\section{POGIL 结合 MOOC 模式运用于医学研究生教育的策略}

尽管 POGIL-MOOC 教学引入医学研究生创新教育具有其独有的优势, 但使其契合我国医学 研究生教育的特点, 也不能完全照搬国外模式, 还需要具体问题具体分析, 以确保充分发挥 POGIL-MOOC 教学的积极作用, 培养学生的创新能力, 促进我国医学的发展。

3.1. 科学化分组

在以导师负责制为导向的医学研究生培养中, 引入 POGIL 教学, 科学化分组是第一个关键步 骤。尽管医学研究生入学水平差异较小, 基础知识水平比较一致, 但每个学生的思维能力、 自主能力以及性格等均存在很大差异。一个合理的研讨小组, 在自由选择的基础上, 更应充 分考虑每个学生的特色, 并考虑其将在讨论组中扮演的角色, 实现性格或能力的互补, 以相 互促进提升自我; 每组成员不易过多, 每组 4-5 人; 研讨小组可设主持人、组长、记录员等 角色, 各角色可定期轮换; 组长应确保每位小组成员切实参与到每次的讨论中, 引导成员根 据提前已经准备的资料，积极发表自己的意见。

3.2. 及时更新关键知识点

每次研讨会的主题应根据研究生水平的提升而变化, 即可分为初级、中级、高级阶段。首先, 巩固本专业基本医学知识; 其次, 针对重难知识点展开讨论; 最后, 围绕国际新技术新进展 进行知识拓展。其中, 导师扮演的角色是学习推动者, 观察学习进展, 解决学生学习需要。 定期每周一到两次研讨会, 导师提前一周选定主题, 由学生围绕主题自主查阅文献, 提取数 据, 分析问题, 得出自己的结论, 并提交一份纸质研究报告。在研讨会后, 学生可根据研讨 过程中提出的分歧与争议，修订完善自己的研究报告。从单一的了解某疾病的临床症状、基 本治疗, 转向探索疾病的发病机制、是否有更优的治疗方案等等。这对提升研究生的逻辑思 维能力, 培养科研思维医学意义重大。相对于其他人文社科类学科其趣味性较低, 内容相对 枯燥。MOOC 教学的引入正好弥补这一问题。慕课微视频表现形式多样, 对各个医学问题可 以用 Flash 动画甚至是三维图像来呈现, 能够最大限度地激发学生的兴趣, 给学生一个直观 印象, 更易于学生消化理解。

3. 3. 构建多样化慕课平台

POGIL 教学尽管以研讨会的形式开展, 但交流形式不局限于研讨会上, 应线上线下同步学, 相辅相成。可利用新型多媒体如微信群等及时分享查阅的领域内最新进展, 推荐 MOOC 平台 中的精彩视频。同时, 在共享现有国内外慕课平台的基础上, 应自主构建多样化医学研究生 培养慕课平台。在慕课课程的准备上, 课程设置丰富化, 杜绝照本宣科产生的无趣感, 导致 学生对慕课丧失兴趣。医学院校可以通过赛课、问卷调查等方式, 发掘讲课生动, 富有吸引 力的教员。通过 “引进来, 走出去”, 邀请知名教授或网络红人教师录制慕课微视频, 最大限 度的激发学生探索热情。借助 MOOC 平台, 学生在思考中产生的疑问, 可以与名家大师探讨, 实现自我知识储备的提升。同时, 通过网络技术的升级, 可以实现校内高速上网, 甚至可以 在校内实现全覆盖的 WiFi。网络共享才能为随时随地随心的慕课学习创造有利条件。

3. 4. 效果评估体系多元化

医学研究生教育始终处于知识发展和转型的最前沿, 它对知识变革的敏感性要远远超过其他 层次的教育。结果往往不是最重要的评价指标, 更多应注重学生创新思维能力的提升。在评 估 POGIL-MOOC 教学模式的效果时, 应注重评估体系多元化, 不以单纯的笔试作为最终方 案 [13]。评估体系主要分为两个方面, 一是理论水平评价; 二是实操能力评价。理论评价可 依靠案例分析、综述写作、前沿文献报告、课题设计等结合传统的笔试进行综合理论水平评 估。实操能力评价可通过临床分管病人及实验进展检查评价医学研究生临床技能及实验技能。 为培养具有创新思维能力的医学研究生, 多元化的评价体系是关键。 


\section{4. 潜在的挑战及对策}

从增量式发展向转型式发展过程中, 尽管 POGIL-MOOC 教学模式可弥补以往教学模式在创 新性人才培养中的不足, 但它在医学研究生教育的具体实施过程中也面临挑战。这些亟待解 决的问题和挑战将成为未来医学研究生教育改革的研究热点。

4. 1. 导师要求高

POGIL-MOOC 教学模式减少了导师们的单一重复性劳动, 但对导师提出了更高的要求, 需要 兼具丰富的临床工作经验和极强的科研能力，有责任、有思维、懂设计的专业教育者 [14]。 这就需要导师投入比传统教学多几倍的精力。医学研究生的导师们大多需兼顾临床工作与科 研教学工作, 本身剩余时间有限, 对 POGIL-MOOC 教学中学生的引导需要花更大的精力, 无疑对导师们是极大挑战。一方面, 导师们要不断提升自我的能力, 做一个切实指导学生发 散思维的引导者; 另一方面, 成立导师组, 导师组成员 3-5 名, 囊括不同专业导师, 实现学 科交叉的同时，也避免因导师工作太忙而疏忽学生。

4.2. 教学持久性难以把控

精彩的课程内容, 良好的用户体验是保证教学质量的关键因素。研究生教育以是自主学习为 主导, 教学效果的持久性受学生的自学能力及自制力影响 [15]。研讨小组成员如果不严格按 照要求, 提前围绕主题展开探索, 独立思考, 自主学习, 那么 POGIL 教学就毫无价值。同时 MOOC 作为大型开放式网络课程, 整个教学过程都是通过网络来完成, 那么如何杜绝 “替学” 或 “作弊” 现象, 如何把控课程质量以及评估教学效果。为保证教学效果的持久性和有效性, 加强学生的引导和监督是基本要求; 作为研讨小组组长和导师应加强小组成员自主学习能力 的监督, 养成良好的自主学习习惯。除加强监督管理外，需要积极探索基于 “慕课” 的多模 态教学策略, 激发学生学习热情, 培养创新思维。在 POGIL-MOOC 教学过程中可引入游戏 策略，吸引主动投入学习。

\section{5. 结论}

在教学国际化的进程中, 教师是主导, 学生是主体, 课程设置是根本, 教学模式要更新, 考 核方法需多元化。POGIL 联合 MOOC 的教学模式契合了医学研究生创新性培养要求, 符合现代 生物-医学-心理学的医学模式, 弥补了现有 “本科化” 教学模式的不足, 为培养 “产学研” 一 体化的新型医学人才提供了方向。尽管如此, 目前的研究生培养课程无论在实施的深度或广 度, 还存在着不少的问题, 要继续深入学习国外的先进教学理念和方法, 逐步建设具有国际 化视野的高水平师资队伍, 形成研究生课程教学国际化体系, 致力于培养理论和实践并重、 具有创新能力和国际视野的医学新型研究生人才。

\section{6. 致谢}

本文感谢以下基金的资助：中国学位与研究生教育学会研究课题（B2-2015Y0501-022); 重庆 市研究生教育教学改革研究项目 (yjg143006); 重庆市高等教育学会高等教育科学研究课题 (CQGJ15161C); 全军学位与研究生教育研讨会研究课题 (YJZX14D23)

\section{参考文献}

[1] 刘贵华, 孟照海. 论研究生教育的发展逻辑 [J]. 教育研究. 2015, 36 (1) : 66-74.

[2] Collins FS, Varmus H. A new initiative on precision medicine[J]. New England Journal Of Medicine. 2015,372(9):793-795.

[3] Moog R. Process oriented guided inquiry learning: Washington University Libraries; 2014. 
[4] Pappano L. The Year of the MOOC[J]. The New York Times. 2012,2(12):2012.

[5] Alraimi KM, Zo H, Ciganek AP. Understanding the MOOCs continuance: The role of openness and reputation[J]. Computers \& Education. 2015,80:28-38.

[6] Moore C, Black J, Glackin B, et al. Integrating Information Literacy, the POGIL Method, and iPads into a Foundational Studies Program[J]. The Journal of Academic Librarianship, 2015, 41(2): 155-169.

[7] 李中文, 吴涛, 汤必奎. “慕课” 应用于医学细胞生物学教学改革探讨 $[\mathrm{J}]$. 继续教育. $2015(2): 43-44$.

[8] 王丽梅, 姜泓. 医学研究生教育面临的问题与对策 [J]. 中国医药导报. 2015, 12 (7) : 129-131.

[9] Hu HH, Shepherd TD. Using POGIL to help students learn to program[J]. ACM Transactions on Computing Education (TOCE). 2013,13(3):13.

[10] Moog RS, Spencer JN, Straumanis AR. Process-oriented guided inquiry learning: POGIL and the POGIL project[J]. Metropolitan Universities. 2015,17(4):41-52.

[11] Mouratev G, Howe D, Hoppmann R, et al. Teaching medical students ultrasound to measure liver size: comparison with experienced clinicians using physical examination alone[J]. Teaching And Learning In Medicine. 2013,25(1):84-88.

[12]Breslow L, Pritchard DE, DeBoer J, et al. Studying learning in the worldwide classroom: Research into edX's first MOOC[J]. Research \& Practice in Assessment. 2013,8:13-25.

[13] Myers T, Monypenny R, Trevathan J. Overcoming the glassy-eyed nod: An application of process-oriented guided inquiry learning techniques in Information Technology[J]. Journal of learning design. 2012,5(1):12-22.

[14] Stewart DW, Brown SD, Clavier CW, et al. Active-learning processes used in US pharmacy education[J]. American Journal Of Pharmaceutical Education. 2011,75(4):68.

[15]Pierce R, Fox J. Vodcasts and active-learning exercises in a "flipped classroom" model of a renal pharmacotherapy module[J]. American Journal Of Pharmaceutical Education. 2012,76(10): 196.

\section{Acknowledgement}

This research was financially supported by the fund of the Chinese Society of Academic Degrees and Graduate Education (B2-2015Y0501-022); Graduate Education Reform fund of Chongqing (yjg143006); Educational Scientific fund of Chongqing Association of Higher Education (CQGJ15161C); and the project of Academic Degrees and Graduate Education Seminar (YJZX14D23).

\section{References}

[1] Guihua Liu, zhaohai meng. The development logic of graduate education [J]. Educational Research. Vol. 36 (2015), No. 1, pp. 66-74. (in Chinese)

[2] Collins FS, Varmus H. A new initiative on precision medicine[J]. New England Journal Of Medicine. Vol. 372 (2015), No. 9, pp. 793-795.

[3] Moog R. Process Oriented Guided Inquiry Learning[M]: Washington University Libraries, 2014. 
[4] Pappano L. The Year of the MOOC[J]. The New York Times. Vol.2 (2012), No. 12, pp. 2012.

[5] Alraimi KM, Zo H, Ciganek AP. Understanding the MOOCs continuance: The role of openness and reputation[J]. Computers \& Education. Vol. 80 (2015), pp. 28-38.

[6] Moore C, Black J, Glackin B, et al. Integrating Information Literacy, the POGIL Method, and iPads into a Foundational Studies Program[J]. The Journal of Academic Librarianship, Vol. 41 (2015), No. 2, pp. 155-169.

[7] Zhongwen Li, Tao Wu, Bikui Tang. Application of MOOC to medical cell biology [J]. continuing education, (2015), No.2, pp. 43-44. (in Chinese)

[8] Limei Wang, Hong jiang. The problem and countermeasure of medical graduate education. China Medical Herald. Vol.12 (2015), No.7, pp. 129-131. (in Chinese)

[9] $\mathrm{Hu} \mathrm{HH}$, Shepherd TD. Using POGIL to help students learn to program[J]. ACM Transactions on Computing Education (TOCE). Vol. 13 (2013), No.3, pp.13.

[10] Moog RS, Spencer JN, Straumanis AR. Process-oriented guided inquiry learning: POGIL and the POGIL project[J]. Metropolitan Universities. Vol.17 (2015), No.4, pp. 41-52.

[11] Mouratev G, Howe D, Hoppmann R, et al. Teaching medical students ultrasound to measure liver size: comparison with experienced clinicians using physical examination alone[J]. Teaching And Learning In Medicine. Vol. 25 (2013), No.1, pp. 84-88.

[12]Breslow L, Pritchard DE, DeBoer J, et al. Studying learning in the worldwide classroom: Research into edX's first MOOC[J]. Research \& Practice in Assessment. Vol.8 (2013), pp. $13-25$.

[13] Myers T, Monypenny R, Trevathan J. Overcoming the glassy-eyed nod: An application of process-oriented guided inquiry learning techniques in Information Technology[J]. Journal of learning design. Vol. 5 (2012), No.1, pp.12-22.

[14] Stewart DW, Brown SD, Clavier CW, et al. Active-learning processes used in US pharmacy education[J]. American Journal Of Pharmaceutical Education. Vol. 75 (2011), No.4, pp.68.

[15]Pierce R, Fox J. Vodcasts and active-learning exercises in a "flipped classroom" model of a renal pharmacotherapy module $[\mathrm{J}]$. American Journal Of Pharmaceutical Education. Vol. 76 (2012), No.10, pp. 196.

作者简介: 张洪 (1990-), 女, 重庆, 研究实习员, 主要研究方向为医学高等教育改革, E-mai1: annicq@163. com;

罗阳（1979-），男，重庆，副教授、副主任医师、博士生导师（第三军医大学/ 西南大学/中国工程物理研究院), 致力于医学研究生教育教学改革与新型教学模式探索研究, E-mail: luoyang@tmmu. edu. cn; 入选 “万人计划” 中组部青年拔尖人才、重庆市杰出青年基 金、人社部留学人员择优资助、重庆市五四青年奖章, 承担国家级、省部级及军队课题 16 项; 获国家科技进步二等奖、“十一五”军队重大科技成果奖等奖项 11 项。以第一或通讯作者发 表论文 100 余篇，其中 SCI 论文 17 篇（IF>10 的 2 篇，IF>5 的 9 篇），获国际 PCT 专利 3 件 （美国、英国、澳大利亚），国家发明专利 14 件，实用新型专利 2 件，软件著作权 1 件。 